\title{
Pseudo-Elliptic Bandpass Filter with Sub-Harmonic Stubs
}

\author{
J.A.G. Malherbe
}

\begin{abstract}
A simple bandpass filter that approximates equal ripples in both the pass and stop bands, is described. The filter comprises of two stubs, resonant at one half and one quarter of the centre frequency, respectively, and a series overlay capacitor. The filter has a performance comparable to that of a third order elliptic function filter and has a practical bandwidth of $16-34 \%$.
\end{abstract}

Introduction: Cauer (elliptic function) filters have transmission zeros at real frequencies; in bandpass transmission line filters they occur on either side of the centre frequency. The zeros are conventionally created by means of Foster or Brune sections and their design procedures are widely described [1]. In non-conventional Cauer filters, authors have variously made use of tapped half-wave resonators as well as stubs resonating either side of the passband to create additional transmission zeros [2], [3] in order to improve filter selectivity. Tapped and resonating stubs have also been widely applied to a variety of other filter structures, such as coupled lines, quarter-wave sections [4] - [6] as well as hairpin lines [7]. Cross coupling is traditionally used for the creation of additional zeros [7].

A completely different approach to creating additional transmission zeros (in that case for a bandstop filter) was taken in [8], where two sub-harmonic resonant stubs (SHS) were made to resonate at one half and one quarter of the centre frequency, respectively. In this paper, the principle of sub-harmonic stubs is applied to a bandpass structure of extreme simplicity, that realizes bandpass filters of $17 \%$ to $38 \%$ relative bandwidth. The SHS filter performance is compared to that of a Cauer filter.

Filter Structure: In the SHS microstrip filter with centre conductor pattern shown in Fig. 1, the stub $Z_{1}$ resonates at one quarter of the filter centre frequency $f_{0}$, i.e. at $f_{01}=f_{0} / 4$, with $\ell_{01}=\lambda_{0}$, contributing two transmission zeros above and below the centre frequency.

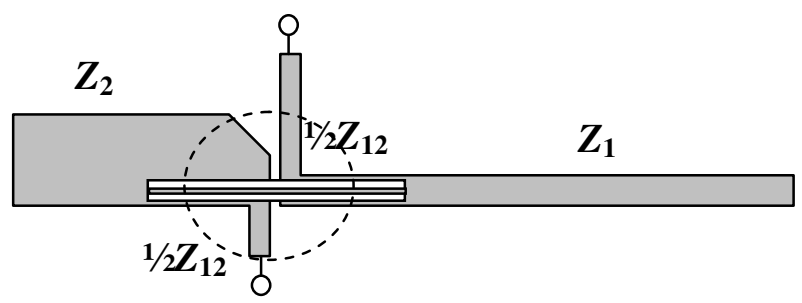

Fig. 1 Layout of the SHS filter.

The stub $Z_{2}$ resonates at $f_{02}=f_{0} / 2$, with a length of $\ell_{02}=\lambda_{0} / 2$, and contributes one zero either side of the centre frequency. The two stubs are connected by a series open 
circuited stub of characteristic impedance $Z_{12}$, resonating at $f_{0}$, length $\ell_{0}=\lambda_{0} / 4$. This stub is split into two equal series stubs.

Fig. 2 shows detail of the connection of the stubs; the stub $Z_{1}$ is formed between $a$ and the earth plane, and stub $Z_{2}$ between $b$ and the earth plane. The series open circuit stub consists of the two sections connected as overlays between $a$ and $c$, and $b$ and $d$, respectively. The two overlay stubs are connected in series between $d$ and $c$. The overlay lines are realized by mounting a narrow strip of the microstrip substrate employed, with the microstrip line on top of it, over the sub-harmonic stubs. They are substantially wider and longer than the overlay lines, and can thus accommodate them as if they were internal series lines.

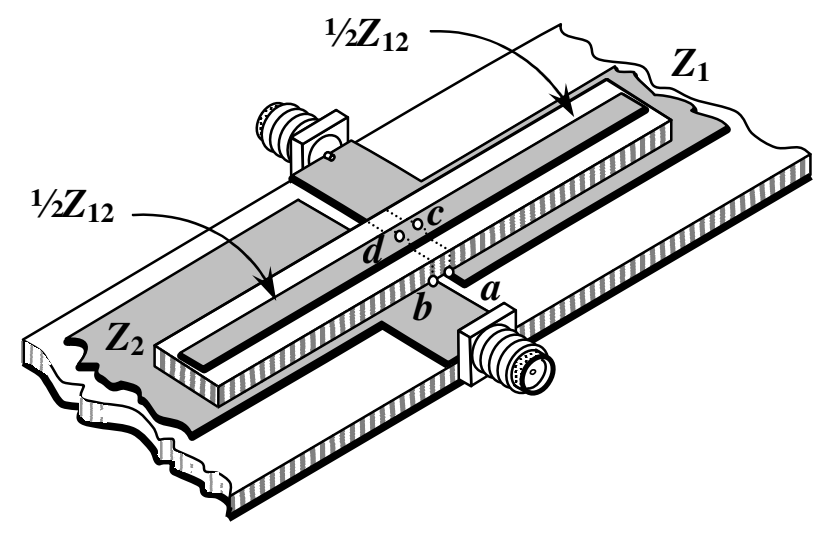

Fig. 2. Expanded view of the stub connections.

Note that the two stubs, each of impedance $1 / 2 Z_{12}$, are connected in series with each other and the two shunt sub-harmonic stubs, yielding the equivalent circuit shown in Fig. 3.

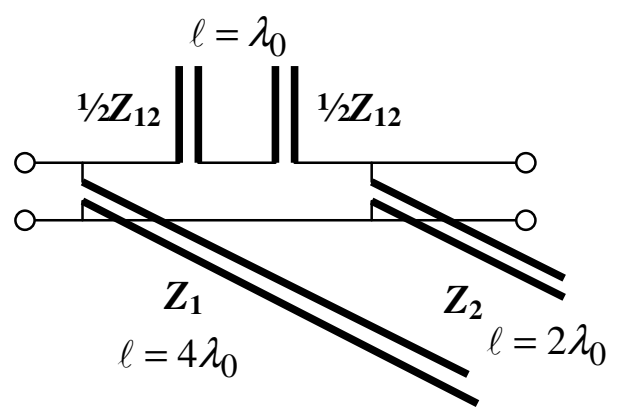

Fig. 3. Equivalent circuit of the SHS filter.

The impedance $Z_{12}$ strongly influences the bandwidth of the filter, as well as the passband VSWR. As the bandwidth is reduced, the impedance levels of $Z_{1}$ and $Z_{2}$ will rise in order to maintain acceptable performance; as the bandwidth is decreased, the passband VSWR is reduced, while the stopband isolation increases. At the widest bandwidth the stopband attenuation is approximately $20 \mathrm{~dB}$ with a passband return loss of $10 \mathrm{~dB}$. Acceptable performance is very easily obtained by repeated analysis. 
Comparison with Elliptic Function Response: The performance of a SHS bandpass filter with $Z_{1}=40 \Omega, Z_{2}=14 \Omega$, and $Z_{12}=250 \Omega$ was evaluated and compared to that of a third order Cauer filter designated C325 [9] with a $0.28 \mathrm{~dB}$ passband ripple and $-26.8 \mathrm{~dB}$ stopband level. The Cauer filter has two shunt shorted stubs of $413 \Omega$ as end sections with a central shunt connected series Foster section comprising of a unit element of 62.9 $\Omega$ and open circuit stub of $271 \Omega$.

The of $S_{21}$ and $S_{11}$ responses for the two filters are compared in Fig. 4. Except for the passband return loss, the stopband isolation and rate of cutoff are comparable.

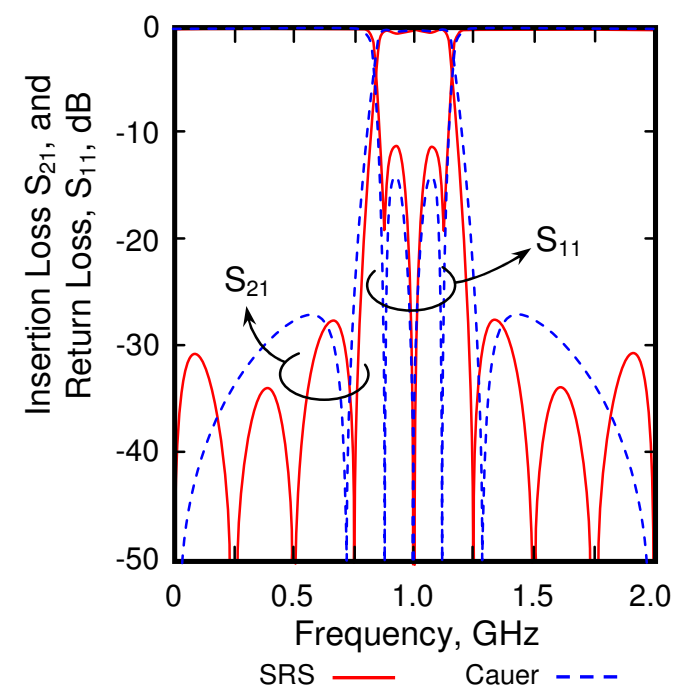

Fig. 4. Insertion loss and return loss of the SHS and Cauer filter.

Measured Results: A prototype SHS filter was constructed in microstrip on RT Duroid 5880 with a dielectric thickness of $1.57 \mathrm{~mm}$, relative dielectric constant of 2.2 and loss tangent $\delta=0.0009$. The series stub was constructed of a strip of the same RT Duroid, 5 $\mathrm{mm}$ wide and $98 \mathrm{~mm}$ long, and bonded to the top of the sub-harmonic stubs with cyanoacrylate adhesivec. The measured insertion loss and return loss is shown in Fig. 5. Both measured insertion and return loss show excellent agreement with the calculated values for the prototype filter.

Conclusion: The sub-harmonic resonant stub filter exhibits a pseudo-elliptic function response that compares well with the third order elliptic function filter, but which is substantially simpler to construct, and smaller. Due to the transmission zeros at real frequencies being determined by the centre frequency of the filter, the applicable bandwidth is limited to between $16 \%$ and $34 \%$. The first spurious passband occurs at 3 $f_{0}$, as for true quarter-wave transmission-line filters. 


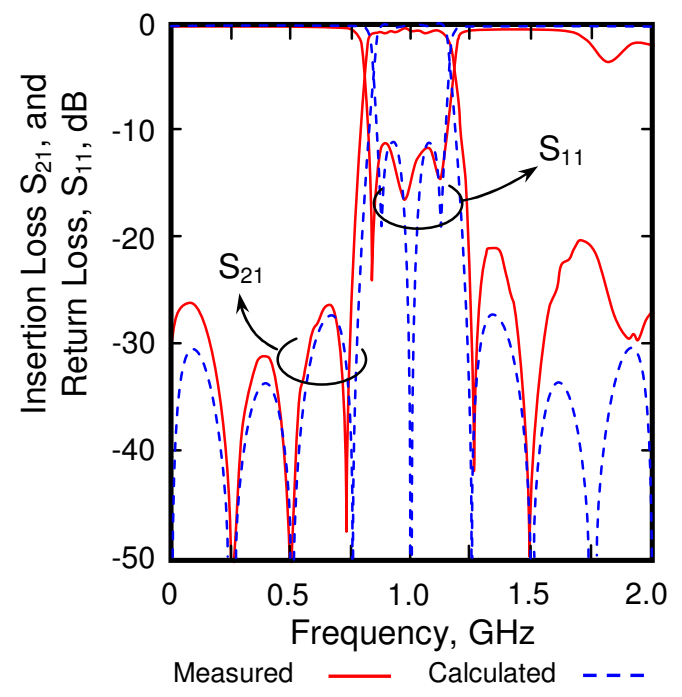

Fig. 5. Calculated and measured insertion loss and return loss of the SHS filter.

\section{REFERENCES}

[1] Malherbe, J.A.G., "Microwave Transmission Line Filters" (Artech House, Dedham, Mass. 1979).

[2] Wada, K., and Awai, I., "Heuristic models of half-wavelength resonator bandpass filter with attenuation poles", Electron. Lett., 1999, 35, (5), pp. 401402.

[3] Gan, H., Lou, D., and Yang, D., "Compact Microstrip Bandpass Filter With Sharp Transition Bands", IEEE Microw. Wirel. Compon. Lett., 2006, 16, (3), pp.107-109.

[4] Zhu, L., and Menzel, W., "Compact Microstrip Bandpass Filter With Two Transmission Zeros Using a Stub-Tapped Half-Wavelength Line Resonator", IEEE Microw. Wirel. Compon. Lett., 2003, 13, (1), pp. 16-18.

[5] Hung, C.-Y., Weng, M.-H., Yang, R.-Y., and Su, Y.-K., "Design of the Compact Parallel Coupled Wideband Bandpass Filter With Very High Selectivity and Wide Stopband", IEEE Microw. Wirel. Compon. Lett., 2007, 17, (7), pp. 510-512.

[6] Duong, T.H., and Kim, I.S., "Steeply Sloped UWB Bandpass Filter Based on Stub-Loaded Resonator", IEEE Microw. Wirel. Compon. Lett., 2010, 20, (8), pp. 441-443.

[7] Lee, S.-Y,. and Tsai, C.-M., "New Cross-Coupled Filter Design Using Improved Hairpin Resonators", IEEE Trans. Microw. Theory Tech., 2000, 48, (12), pp. 2482-2490.

[8] Malherbe, J.A.G., and Reid, C., "Double Resonant Stub Bandstop Filter with Pseudo-Elliptic Response", Electron. Lett., 2010, 46, (7), pp. 508-509.

[9] SAAL, R.: "Handbook of Filter design", (AEG-Telefunken, Berlin, 1979).

\section{Authors' affiliation:}

J.A.G. Malherbe (Department of Electrical, Electronic and Computer Engineering, University of Pretoria, Pretoria 0002, South Africa, jagm@up.ac.za) 